\title{
Germanica
}

\section{Le thème du voyage intérieur dans les contes de Jeanna Oterdahl*}

Die innere Reise. Ein Motiv in den Märchen von Jeanna Oterdahl

Lena Kåreland

\section{(2) OpenEdition}

\section{Journals}

Édition électronique

URL : http://journals.openedition.org/germanica/1283

DOI : $10.4000 /$ germanica. 1283

ISSN : 2107-0784

Éditeur

Université de Lille

Édition imprimée

Date de publication : 1 janvier 1992

Pagination : 125-141

ISSN : 0984-2632

\section{Référence électronique}

Lena Kåreland, "Le thème du voyage intérieur dans les contes de Jeanna Oterdahl* », Germanica [En ligne], 11 | 1992, mis en ligne le 07 février 2014, consulté le 06 octobre 2020. URL : http:// journals.openedition.org/germanica/1283; DOI : https://doi.org/10.4000/germanica.1283

Ce document a été généré automatiquement le 6 octobre 2020.

(C) Tous droits réservés 


\title{
Le thème du voyage intérieur dans les contes de Jeanna Oterdahl*
}

\author{
Die innere Reise. Ein Motiv in den Märchen von Jeanna Oterdahl
}

\author{
Lena Kåreland
}

1 Parmi les nombreuses femmes écrivains que connut la littérature suédoise au début du xxe siècle, Jeanna Oterdahl est une de celles qui apportèrent une contribution importante à la littérature enfantine. En grande partie oubliée aujourd'hui, son œuvre mérite néanmoins que l'on s'y attarde.

2 Jeanna Oterdahl (1879-1965) était la fille d'un officier de Uddevalla, en Suède occidentale. Elle fit ses études à l'Institut pédagogique royal de Stockholm de 1898 à 1901. À l'époque cette école était le foyer de l'intelligentsia féminine. Selma Lagerlöf avait été parmi ses élèves peu de temps auparavant. Il était rare alors que des femmes suivent des études supérieures. Entre 1901 et 1935 Jeanna Oterdahl fut professeur dans un lycée de jeunes filles à Göteborg. Parallèlement, elle commença à écrire et à donner des conférences. Elle exerça également jusque vers 1960 une activité de critique littéraire spécialisée dans les livres pour enfants. L'œuvre de Jeanna Oterdahl, est marquée par sa vision chrétienne du monde. Elle participa au mouvement quaker, et elle a écrit de nombreux livres religieux, ainsi que des psaumes.

3 La majeure partie de son œuvre s'adresse cependant aux enfants. Elle publia contes, récits, poèmes et livres pour la jeunesse. Elle fit ses débuts en 1901 avec un recueil de poèmes, Solhult. Le livre était illustré par Elsa Beskow, célèbre peintre et auteur de livres pour enfants, qui devait également illustrer le recueil de poésie Blommornas bok (Le Livre des fleurs) en 1905 et Dags visor (Les chansons de Dag) en 1911. La poésie d'Oterdahl se définit par une forme simple et maîtrisée. On y rencontre ce sentiment de la nature et ce nationalisme romantique qui furent une des caractéristiques de l'époque.

4 Avec la publication de son roman Inger Skram, en 1919, Jeanna Oterdahl s'adresse aussi à un public plus vaste que celui de la littérature enfantine. Dans sa littérature pour jeunes filles il faut surtout mentionner Skolflickor (Les lycéennes) en 1924, et le cycle 
Helga Vilhelmina en 1933. Ce dernier s'inscrit dans le courant du roman prolétarien et du roman de formation : il raconte la vie d'une petite fille pauvre, assoiffée de savoir et décrit la folkhögskola suédoise, son milieu et sa forme scolaire particulière. Skolflickor est un recueil de nouvelles dont l'action se déroule dans un lycée de jeunes filles. L'œuvre d'Oterdahl comprend également un certain nombre de biographies destinées à la jeunesse, entre autre celles de Selma Lagerlöf et de H.C. Andersen.

5 Ses contes pour enfants se caractérisent souvent par leur ton mélancolique et leur prédilection pour les sujets tristes. Cela lui fut reproché par des critiques littéraires contemporains, qui trouvèrent certains de ses contes par trop sinistres.

6 La vie et l'œuvre de Jeanna Oterdahl sont à beaucoup d'égards typiques pour son époque. Elle fut l'une de ces nombreuses femmes cultivées, issues de la bourgeoisie, qui firent de la littérature enfantine leur domaine de prédilection.

7 Vers 1900 la littérature enfantine connaît son premier essor en Suède. La spécificité de l'enfant est reconnue et on cherche à améliorer ses conditions de vie. Critiques et intellectuels insistent sur la nécessité de proposer aux enfants des livres d'une haute qualité littéraire et artistique. Beaucoup condamnent en même temps la littérature enfantine moralisante et tendancieuse qui avait prévalu jusque là. Parallèlement, des actions sont entreprises en faveur de l'instruction populaire, et des efforts engagés pour donner aux enfants les moins favorisés un meilleur accès au livre. L'esprit de l'époque est marqué par la foi au progrès, par l'idéalisme esthétique et par un sentiment national, trait commun de toute une génération. On cherche par ailleurs à supprimer les frontières entre littérature pour enfants et littérature pour adultes. Le conte qui était déjà un genre important, obtient droit de cité non seulement dans la chambre des enfants, mais aussi au salon ${ }^{1}$.

8 La conception littéraire des années 1890 , qui préconisait l'imagination et valorisait le romantisme national, contribua à augmenter le prestige du conte. Des écrivains tels que Selma Lagerlöf et Verner von Heidenstam, créèrent avec Nils Holgerssons underbara resa (Le merveilleux voyage de Nils Holgersson) et Svenskarna och deras hövdingar (Les Suédois et leurs chefs) des œuvres qui entrèrent à l'école primaire et furent lus par tous les enfants de Suède. Par ailleurs, la progression du mouvement ouvrier social-démocrate avait produit des conditions sociales nouvelles. De plus larges fractions de la société exigèrent d'être associées au fonctionnement et aux bienfaits de la société ${ }^{2}$.

Il importe cependant de constater que l'on ne peut guère parler d'une irruption de la modernité dans la littérature pour enfants. L'idéologie véhiculée par la majorité des livres pour enfants, consistait à faire comprendre aux jeunes où était leur place dans la société et à les persuader de s'y maintenir avec gratitude. L'humilité, l'acceptation de sa situation, l'assiduité au travail étaient les idéaux le plus souvent mis en avant. La propagande religieuse et patriotique y tenait une place considérable ${ }^{3}$. Les livres du début du XXe siècle, qui sont encore lus et appréciés de nos jours et qui sont devenus des classiques, ceux d'Elsa Beskow ou d'Ottilia Adelborg, ou encore d'Ivar Arosenius, ne sont guère représentatifs de la littérature enfantine de l'époque. Ces œuvres d'une très haute qualité littéraire constituaient le sommet d'une pyramide dont la base était une littérature à forte tonalité moralisante et didactique et, somme toute, assez médiocre. Pendant la période 1880-1910 le nombre des publications de contes avait considérablement augmenté ${ }^{4}$. Beaucoup de ces contes étaient publiés dans des magazines pour enfants, publications de Noël, et touchaient par ce biais un large public ${ }^{5}$. C'était la première grande période du conte littéraire suédois. Celui-ci, dont 
l'évolution a été décrite dans une thèse française ${ }^{6}$, trouve une de ses origines dans le conte populaire. Mais la légende était également une source d'inspiration importante ${ }^{7}$. Le conte, qu'il soit littéraire ou populaire, était considéré par les critiques comme étant la lecture la plus adaptée pour l'enfant. Ellen Key, une féministe célèbre, qui fut à l'origine du mouvement en faveur de l'éducation artistique en Suède et qui formula le concept «skönhet for alla» (la beauté pour tous), fut un grand défenseur du conte, un genre dans lequel s'illustreront surtout des écrivains féminins ${ }^{8}$.

10 Jeanna Oterdahl publia au cours de sa vie une quinzaine de recueils de contes. Beaucoup d'entre eux parurent d'ailleurs à la fois sous forme de livre et dans des magazines. Son style est à la fois limpide et discret. La composition des contes est resserrée et présente la même simplicité de structure que le conte populaire. Parfois, on note un lien évident avec l'époque et une problématique d'actualité, la question de l'émigration par exemple. Le plus souvent cependant, ces contes se caractérisent par leur intemporalité et leur universalité, un trait qu'ils ont en commun avec le conte populaire. Sur le plan formel également, les contes d'Oterdahl suivent la plupart du temps le schéma du conte populaire. Ils sont volontiers introduits par la phrase classique: "Il était une fois...» Le nombre trois y est fréquemment utilisé et le surnaturel y est souvent présent sous forme d'animaux dotés du langage, d'oiseaux, de trolls ou de lutins. En règle générale ils se caractérisent par leur manque d'éléments dramatiques. Les ennemis extérieurs y sont rares. Les problèmes sont plutôt d'ordre existentiel ou moral. Les héros du conte livrent un combat intérieur, où le Bien lutte contre le Mal. Il s'agit de montrer comment se trempe le caractère du héros et comment se développe sa personnalité. Le message des contes s'accorde avec le code moral en vigueur. Oterdahl parvient à intégrer la morale dans l'intrigue. Il s'agit rarement d'une morale centrée sur un comportement extérieur. Le problème éthique mis en évidence par Oterdahl, se situe sur un plan plus profond et concerne davantage la transformation d'une disposition d'esprit, d'un trait de caractère. Un autre aspect important est le rapport de l'homme avec la nature'. Parfois les contes sont proches de la légende et rappellent entre autre les célèbres Kristuslegender (Légendes du Christ) de Selma Lagerlöf, publiées en 1904.

11 Le conte littéraire a été décrit comme l'intermédiaire entre l'imitation du conte populaire, le récit romantique en prose et le conte moralisant ${ }^{10}$. Cette définition rend très bien compte de sa particularité et peut également s'appliquer aux contes de Jeanna Oterdahl.

\section{Le pouvoir de l'amour maternel}

12 Le conte «Den bergtagnas dotter» («La fille de la femme ensorcelée par les lutins ») du recueil du même titre (1922) est construit sur un thème récurrent, mais remanié par l'auteur. C'est un bon exemple de la pluralité des niveaux de lecture qu'offrent généralement les contes d'Oterdahl. L'intrigue qui tient le lecteur en haleine se dédouble d'un drame spirituel : «le voyage intérieur ».

Dans ce conte il y a un parallélisme entre le thème de l'enlèvement et celui de l'enfant substitué. La substitution d'un petit troll à un enfant, ou l'abandon par les trolls d'un de leurs rejetons pour qu'il soit élevé par les hommes, est un thème célèbre dans les légendes ${ }^{11}$. Le récit d'Oterdhal commence donc avec la description d'une jeune mère qui vient de donner le jour à une petite fille. La paix et le bonheur du foyer contrastent 
avec la nuit noire à l'extérieur. Dehors, la tempête gronde et le vent siffle. La lune à moitié cachée derrière les nuages déchirés, jette parfois une lumière blanche à travers les fenêtres. La mère attend le retour du père pour se réjouir avec lui de la naissance du nouveau-né. Puis la lune disparaît un instant derrière un nuage, la mère entend la porte grincer et des bruits de pas. Quelqu'un s'approche du berceau. Terrifiée, la mère n'ose rien dire et une sueur d'angoisse couvre son front. Lorsqu'un rayon de lune éclaire de nouveau les carreaux de la fenêtre, elle aperçoit l'intrus.

Elle voyait une jeune femme, blonde au teint clair.

La femme portait un habit d'une facture singulière et

le clair de lune faisait briller les pierres de son diadème,

les chaînettes et les boucles qui entouraient sa taille.

Son visage révélait une angoisse secrète et sauvage ${ }^{12}$.

Lorsque la mère regarde le berceau pour s'assurer que l'enfant est sain et sauf elle voit deux petites filles au lieu d'une seule. Elles se ressemblent comme deux gouttes d'eau, toutes les deux « aussi menues et vulnérables, ayant autant besoin de ses soins et de son amour l'une que l'autre ». Elle hésite, puis décide de garder les deux enfants, se disant qu'elle saura bien distinguer quelle est la sienne et quelle est celle de l'étrangère. Mieux vaut dire à son mari que les deux enfants sont les leurs. Quand, au matin, les deux valets de la ferme viennent voir les petites dans le berceau, ils racontent l'histoire d'une jeune fille disparue il y a trois ans, avant que la jeune mère ne vienne à la ferme. Tous étaient persuadés alors qu'elle avait été enlevée par les trolls. Les valets sont persuadés que c'est cette jeune fille-là qui venait de déposer dans le berceau l'enfant qu'elle avait eu d'un troll pour qu'il grandisse chez les hommes.

La mère est épouvantée par ce qu'elle vient d'entendre. Est-ce un petit de troll qu'elle doit élever comme s'il était son enfant ? Je chasserai cette créature et la déposerai dans la forêt dès que je pourrai voir que c'est un troll, pense-t-elle. Lorsque son mari rentre, il est tout heureux en découvrant qu'il y a deux petites filles dans le berceau. La mère craint qu'il ne s'aperçoive de quelque chose, mais il n'en est rien et l'homme traite les deux filles avec la même tendresse. Dans son for intérieur elle s'en réjouit, car elle s'est déjà attachée aux deux enfants et ne veut plus s'en séparer.

Les années passent et les filles grandissent. Elles sont très différentes : l'une est blonde aux yeux bleus, d'un tempérament violent, l'autre brune aux yeux gris, d'une nature tranquille et douce. La mère se demande constamment laquelle des deux enfants est la fille des trolls. Elle aime autant l'une que l'autre, mais souffre d'avoir dissimulé la chose à son mari.

17 Un jour, l'homme raconte que, de l'autre côté de la montagne, une famille a trouvé un petit troll dans le berceau du nouveau-né. Leur enfant n'a pas été enlevé, les trolls ont simplement mis un des leurs à côté de lui dans le berceau. Or, les deux enfants se ressemblent tellement que personne ne peut distinguer le petit des trolls de l'enfant des hommes. La mère écoute son mari avec angoisse. Des touffes de poils ne tarderont pas à pousser dans les oreilles du troll et on pourra alors le chasser, déclare l'homme. Un troll reste un troll et aucun homme ne peut s'attacher à un troll, affirme-t-il avec fermeté, lorsque la femme essaie prudemment de lui dire qu'ils aimeront peut-être autant les deux enfants. L'homme se félicite de ce que tout soit normal en ce qui concerne leurs propres enfants, à cent lieues de se douter de la situation réelle.

La femme décide finalement de tout révéler à son mari la veille du quinzième anniversaire des filles. L'homme refuse d'abord de la croire. Il pense qu'elle a perdu la 
raison et que tout est le fruit de son imagination. Ce n'est que lorsque les valets confirment les dires de sa femme qu'il se laisse convaincre. Cependant, il ne réagit pas avec colère, comme l'avait redouté sa femme mais avec une immense tristesse. Comment pourrais-je être heureux maintenant que je sais que l'une d'elles est un troll, explique-t-il ? Pourtant il doit confesser que les deux enfants lui sont également chères.

Peut-être l'amour crée-t-il des liens plus forts que le sang, dit la femme, qui avoue également ne vouloir pour rien au monde revenir en arrière. Sans son intervention, un de leurs enfants aurait été élevé comme un troll parmi les trolls.

Remercions Dieu, qu'il n'en soit pas ainsi, dit l'homme doucement. C'est grâce à toi, Mère, qu'une âme a été sauvée ${ }^{13}$.

Puis ils montent l'escalier et entrent dans la chambre des enfants où les deux sœurs sont endormies. C'est alors qu'ils entendent la porte grincer et le cliquetis des clochettes et chaînettes. La femme ensorcellée se tient à nouveau dans la pièce et se penche comme eux au-dessus du lit des enfants. N'ayez pas peur, dit-elle. Je ne suis pas venue vous dérober quelque chose. Je suis venue vous remercier. Lorsque la femme veut lui demander laquelle des deux filles est la sienne l'homme l'en empêche, et prie l'étrangère de ne rien révéler. Avec un sourire mélancolique la femme ensorcelée répond : «Je sais seulement que la petite graine d'âme qui était en ma fille a poussé et fleuri chez vous ».

«Den bergtagnas dotter» est un conte sous l'emprise du fatum, marqué par une forte tension dans sa construction. L'intrigue a quelque chose d'inexorable comme dans la tragédie grecque. La dimension dramatique et ironique se manifeste notamment en ce que le lecteur détient davantage d'informations que les personnages du conte : par exemple, le mari n'apprendra la vérité sur les deux filles qu'à la fin. Le dialogue entre l'homme et la femme, à propos de l'histoire survenue à la famille de l'autre côté de la montagne, est chargé de tension. L'assurance tranquille de l'homme qui croit que pareille chose ne peut leur arriver s'oppose avec une ironie marquante à l'angoisse de la femme qui va devoir révéler la vérité contre son gré.

21 «Den bergtagnas dotter» est en premier lieu un conte moral à propos de l'amour, singulièrement de l'amour maternel. Les mots clefs du texte sont ceux prononcés par la femme : «l'amour est plus fort que le sang ». En donnant aux deux enfants l'amour et l'attention dont ils ont besoin, les parents démontrent que les forces du Bien triomphent sur celles du Mal. Le conte illustre ainsi l'idée chrétienne de l'amour formulée dans l'adage "fais à autrui ce que tu veux qu'il te fasse ». L'amour maternel donné en exemple n'est pas l'amour maternel aveugle, instinctif, qui ne se porte que sur la progéniture biologique, mais un amour maternel universel qui fait penser aux conceptions de la tendresse maternelle et féminine formulées par Ellen Key, très répandues à l'époque. La sollicitude maternelle doit dépasser la famille, pénétrer la société dans son ensemble et se manifester dans l'attitude devant la vie.

Le thème religieux du conte prend un sens différent à la lumière des idées du mouvement pédagogique réformateur de l'époque.

On était persuadé que l'instruction avait une importance primordiale, y compris pour le développement de la personnalité ${ }^{14}$. L'accès des hommes au savoir, ainsi qu'à l'art et à la littérature, devait leur ouvrir des possibilités de développement quasi illimitées. Avec sa vision idéaliste Oterdahl démontre dans «Den bergtagnas dotter» comment un rejeton du Roi des trolls, symbole des forces négatives et du Mal, peut devenir contre toute attente, un être bon lorsque les conditions sont favorables. Le conte illustre sur ce 
plan le débat sur l'influence de l'hérédité et celle du milieu, et sur les inégalités sociales.

A la différence du conte populaire, c'est le conflit intérieur et moral qui est au cœur du conte d'Oterdahl. La mère est déchirée entre le désespoir d'avoir la progéniture d'un troll dans le berceau et l'amour maternel qui ne fait pas de différence entre les enfants. Sa décision de ne pas révéler la vérité à son mari est source de conflits et elle souffre d'être obligée de mentir. Les luttes intérieures auxquelles s'exposent l'homme et la femme les font progresser et mûrir. Leurs expériences renforcent leur amour et les rapprochent l'un de l'autre alors que dans le conte populaire, les personnages sont plus statiques et n'évoluent ou ne se transforment que rarement. Les personnages décrits dans le conte d'Oterdahl gardent néanmoins un caractère très général. Ils ne sont pas individualisés et n'ont pas de noms. Ils sont présentés comme les archétypes de la mère et du père.

A l'instar du conte populaire, l'histoire est construite sur des contrastes. Le texte comporte les dualismes classiques: l'obscurité et la lumière, le Bien et le Mal, les hommes et les trolls, l'esprit et la nature. Les trolls personnifient la primitivité, la bestialité et le Mal.

\section{La morale du sacrifice}

La victoire sur la solitude intérieure, le développement de l'amour du prochain ou l'abnégation de soi sont des thèmes qui reviennent constamment dans l'œuvre d'Oterdahl ${ }^{15}$. Le conte intitulé «Den hemlighetsfulla trädgården» (« Le jardin secret »), également tiré du recueil Den bergtagnas dotter en est une illustration intéressante. Il peut se lire comme une allégorie sur le but et le sens de la vie humaine. Le conte repose sur une trame qui est caractéristique du genre, celle de l'errance et de la quête ${ }^{16}$. Le personnage principal de «Den hemlighetsfulla trädgården» est une petite fille prénommée, Elsalill, dont la grand-mère lui raconte l'histoire du jardin :

Il faut marcher longtemps, longtemps, raconta grand-mère. Il faut continuer de marcher jusqu'au bout du monde et ce n'est qu'ensuite que l'on atteint le jardin secret. Mais la grille est fermée et ne s'ouvre que si l'on trouve la clef. La clef est petite, toute petite, elle est en or, mais tout le monde ne la trouve pas; et sans clef on ne peut pas entrer. Le jardin secret est le plus beau des jardins, un paradis terrestre. Tout ce qui peut réjouir le cœur des hommes y pousse et tout ce qui apporte guérison et réconfort. Tu peux y remplir ta corbeille de fleurs et de fruits et l'emporter dans le monde et elle ne désemplira jamais ${ }^{17}$.

Il tarde à Elsalill de connaître le jardin secret. En grandissant, elle devient une jeune fille timide et taciturne. Quand la grand-mère meurt elle se sent très seule. Elle pense constamment au jardin et décide finalement de tenter de le trouver. Le chemin est infiniment long. Elsallil rencontre beaucoup de personnes sur sa route et s'efforce d'aider ceux qui souffrent. Un jour cependant elle rencontre un jeune homme et connaît l'amour. Les deux jeunes gens continuent leur route ensemble. Sans qu'ils en soient conscients l'amour les isole de leurs semblables et leur fait oublier les peines et les souffrances des autres. Mais, un jour, le jeune homme disparaît soudain. Elsalill le pleure. "Autour d'elle la lande s'étendait froide et gelée. Le bras protecteur avait disparu». Bientôt cependant, Elsalill redécouvre ses compagnons de route. Elle comprend encore mieux que jadis leur détresse, car elle sait ce qu'est la perte d'un être cher et la souffrance. Elle croit deviner le but de sa quête, le jardin secret. En passant 
devant une petite maison grise elle entend des pleurs et des sanglots. Lorsqu'elle entre dans la maison, elle découvre cinq enfants affamés, debout autour de leur mère, qui gît, morte, sur un tas de vieux chiffons. Elsalill comprend qu'elle doit rester là pour soulager cette misère. Dès lors elle est trop occupée pour avoir le temps de penser à elle-même, elle apprend aussi bien à souffrir qu'à se réjouir avec ses prochains. «Elle vécut dans l'intimité de la détresse et de la joie, car elle comprenait l'une et l'autre ».

Le temps passe et les enfants grandissent. Elsalill elle-même est devenue une vieille femme. Une nuit elle rêve qu'elle a en main la clef d'or. Elle se trouve au milieu du jardin secret et voit sa grand-mère venir à sa rencontre. Je suis enfin arrivée, s'exclamet-elle. La grand-mère sourit et dit que cela fait déjà longtemps qu'elle séjourne dans le jardin secret. Elsalill ne comprend pas bien mais la grand-mère lui dit :

Que cherchais-tu ici? demande-t-elle. Ne voulais-tu pas remplir ta corbeille de belles plantes, de tout ce qui peut aider, donner la joie et le réconfort? Tu ne sais donc pas que tu portes cette corbeille à ton bras depuis bien des années ${ }^{18}$. Lorsque Elsalill se réveille, la clef est effectivement dans sa main. Elle comprend alors qu'elle vit dans ce paradis terrestre auquel depuis son enfance elle a donné le nom de jardin secret. Il se trouve dans son cœur.

Dans Den hemlighetsfulla tràdgarden comme dans Den bergtagnas dotter les événements qui se succèdent sont d'ordre psychologique. Le premier conte décrit, sous forme symbolique, le parcours d'un être humain, sa quête de ce qu'il estime important et de ce qu'il s'est assigné comme but. Le conte couvre toute l'existence de l'héroïne, de la naissance à la vieillesse ; le conte traditionnel, au contraire, relate le plus souvent une succession d'épisodes à un moment précis de la vie du personnage. La question se pose cependant de savoir s'il s'agit bien ici d'un conte, alors que le surnaturel en est absent, ou plutôt d'une légende allégorique.

Le message transmis est l'idée chrétienne du sacrifice, et du renoncement. L'homme doit dépasser le souci de son intérêt personnel et veiller surtout aux besoins de son prochain. Le récit peut être interprété comme une illustration des paroles du Sermon sur la montagne : celui qui donne est plus heureux que celui qui reçoit - et du paradoxe biblique selon lequel celui qui perd la vie la gagnera. Elsalill vit une transformation radicale de sa personnalité. Elle apprend à s'ouvrir à autrui, à sortir de soi et à s'oublier elle-même. Elle développe sa faculté d'aimer son prochain et de compatir aux soucis de ses semblables. Cette transformation spirituelle est présentée sous la forme d'un conte. Comme dans le classique Voyage du pèlerin de Bunyan, la quête du jardin apparaît ici comme une allégorie, de l'effort d'Elsalill pour comprendre son propre combat intérieur et son aspiration à la plénitude et à la vérité. Grâce à la transformation de sa personnalité, elle parviendra à l'harmonie intérieure. Au cours de sa quête elle aura vu la vie humaine sous tous ses aspects, la détresse, la souffrance, mais elle aura aussi connu l'amour.

Lorsque Elsalill rencontre l'homme qu'elle aime, elle tend «à oublier pourquoi elle marchait et dans quel but ». Les deux amants sont tellement absorbés par leur amour qu'Elsalill ne voit plus les hommes qui souffrent. L'amour pour un seul homme empêche Elsalill d'atteindre le jardin paradisiaque qu'elle cherche. Elle est très heureuse en vivant avec l'homme qu'elle aime. Cependant, elle doit renoncer au bonheur de l'amour terrestre pour trouver la clef d'or.

L'expérience de l'amour amène néanmoins Elsalill à une meilleure compréhension de la souffrance d'autrui, et l'aide à l'apaiser: "Ses mains qui touchèrent des membres 
ensanglantés et meurtris étaient plus tendres que jamais, le son de sa voix plus doux et plus profond, l'éclat de ses yeux plus vif ». La morale du sacrifice prêchée dans le conte paraît aujourd'hui assez démodée. Le récit doit naturellement être replacé dans le contexte de l'époque. Le dévouement et l'abnégation étaient des vertus qu'il était important de transmettre aux enfants au début du siècle. Ce à quoi l'homme devait tendre, ce n'était pas le bonheur personnel mais plutôt l'offrande de la joie et du bonheur à son prochain et l'acceptation de son sort. Considéré de notre temps, le texte peut passer pour l'apologie du renoncement à la belle vie. Il faut cependant prendre en considération que la vie d'Elsalill, telle qu'elle est décrite, est riche et pleine de sens, c'est une vie active, tournée vers l'extérieur. La jeune fille réservée, timide et égocentrique qu'elle était devient une vieille femme sage, pleine d'expérience, en harmonie avec elle-même. Sacrifices et privations ne l'ont pas rendue amère.

La morale du sacrifice sur laquelle est construit Den hemlighetsfulla trädgårdem est un thème que l'on retrouve dans plusieurs contes de Jeanna Oterdahl ${ }^{19}$. Il est par exemple particulièrement présent dans «Undret» («Le miracle»). Ce conte, proche de la légende, raconte l'histoire de Master Albrekt qui édifie une église, une demeure pour le Seigneur «suffisamment haute et vaste pour loger toute l'aspiration, toute la joie et toute la douleur contenus dans le cœur des hommes ».

«Undret» présente une philosophie fortement imprégnée par la foi chrétienne de son auteur, ainsi qu'une dimension visionnaire que l'on retrouve dans l'ensemble de l'œuvre de Jeanna Oterdahl. L'idée du sacrifice, l'oubli et le dépassement de soi-même reviennent, sous différentes formes, dans de nombreux contes d'Oterdahl.

\section{Le départ du foyer}

Le conte «Kvistvättens knyte» («Le baluchon du lutin de Kvisten») du recueil Den bergtagnas dotter, raconte la formation intérieure d'une jeune fille. L'introduction situe l'intrigue à la fois géographiquement et historiquement :

Il était une fois un groupe de jeunes qui avaient l'intention de partir pour l'Amérique. Dans toutes les fermes et dans toutes les maisons à Vikdalen, on n'entendait parler que de cela depuis Noël, et plus on en parlait, moins on pouvait résister à la tentation de Tailleurs ${ }^{20}$.

37 Il s'agit donc de l'émigration, un phénomène qui frappa durement la Suède du XIXe siècle et qui fut l'objet de vives controverses à l'époque ${ }^{21}$. Le pasteur a parlé aux jeunes et souligné les risques encourus par les émigrants. "Faire son chemin en Amérique avait-il dit, c'est comme de traverser un marais peu sûr. Il y en a sans doute qui trouveront à poser les pieds sur de bonnes touffes d'herbe, mais nombreux seront ceux qui perdront pied et s'enfoncent dans les profondeurs ».

38 Mais la fièvre de l'Amérique brûle les jeunes gens et leurs parents ne peuvent s'opposer à leurs aspirations et à leur goût de l'aventure.

Snickar-Jons habite une petite maison à la lisière du bois avec sa femme et ses huit enfants. C'est une famille heureuse qui se contente de peu. Mais l'aînée, Märta, qui a seize ans, a été «ensorcelée par tous ces discours sur ce grand et lointain pays de cocagne »; elle a décidé d'émigrer. Les parents le regrettent mais ils n'ont pas réussi à la faire changer d'avis. Ils pensent néanmoins qu'il est dommage que des jeunes gens vigoureux quittent leur pays natal, où l'on a tant besoin de leurs bras. 
Remplir sa tâche là où elle vous avait été confiée, c'était bien cela qui conduisait au bonheur. N'était-il pas dit dans le catéchisme qu'on devait honorer son père et sa mère ${ }^{22}$. thème fréquent dans le conte populaire, mais aussi dans le conte littéraire. Bruno Bettelheim ${ }^{25}$ a souligné l'importance positive du conte sur les jeunes. Il donne le courage de se séparer de ses parents et de prendre son destin en mains. Dans le conte populaire, le héros réussit cette libération. En revanche, dans le conte littéraire suédois de la fin du XIXe siècle le processus est sensiblement plus conflictuel ${ }^{26}$, en raison de la conception morale de l'époque. Chez Oterdahl il est clair que le devoir envers les parents passe avant le rêve de créer son propre avenir. Pour un lecteur moderne le désir de Märta de partir dans le monde est à la fois naturel et compréhensible, alors que dans le conte il est présenté comme égoïste et irréfléchi. Märta est décrite comme « ensorcelée » et ses préparatifs du voyage se passent dans une sorte d'ivresse qui la rend aveugle aux réactions de son entourage. C'est l'apparition du lutin qui la fait réfléchir. Dans une perspective psychanalytique, cet être surnaturel représente la conscience, ou le sur-moi de Märta. Jusque-là, ses agissements étaient dirigés par des pulsions égoïstes. Pendant la marche à travers la forêt s'opère une sorte d'intégration du moi et du sur-moi. Interprété selon la psychologie de Jung, le lutin apparaît comme l'animus de Märta, le côté masculin de la psyché féminine, qui représente souvent le rationnel. En choisissant le rôle de la fille soumise et dévouée, Märta accepte la morale sociale dominante et l'intègre dans sa vie. Elle a triomphé d'elle-même en suivant sa conscience et ses convictions. $\mathrm{Si}$, en revanche, nous interprétons le conte à partir des théories de la psychanalyste Alice Miller, le texte nous offre un exemple de la pédagogie qui étouffe la volonté de l'individu et induit un sentiment de devoir par manipulation ${ }^{27}$. Parallèlement, la faculté d'initiative et de créativité est entravée.

Dans les contes de Jeanna Oterdahl on retrouve souvent les ingrédients du conte traditionnel, mais son objectif est toujours d'ordre didactique et pédagogique. L'auteur met en lumière des processus psychologiques et traite sous forme symbolique de la condition existentielle de l'homme. Des problèmes métaphysiques, la lutte entre le Bien 
et le Mal, la morale du sacrifice et le rôle du héros sont les thèmes fondamentaux, cachés sous les apparences d'une intrigue ordinaire. Leur conception est profondément enracinée dans la tradition romantique. Ces textes offrent bien évidemment une lecture à plusieurs niveaux. Ils expriment l'esprit et les idées d'une certaine époque, tout en illustrant des questions éternelles de l'humanité.

\section{NOTES}

1. - Kåreland Lena, Gurli Linders barnbokskritik, Bonniers, 1977, chapitre III.

2. - Kåreland Lena, «Ellen Key och konstuppfostransidealet», dans Litteraturens vägar. Litteratursociologiska studier tillägnade Lars Furuland', Gidlunds, 1988, p. 190.

3. - Svensson Sonja, Läsning för folkets barn. Folkskolans barntidning och dess förlag 1892-1914, Rabén \& Sjörgen, 1983, p. 163. Voir aussi Tingsten Herbert, Gud och fosterlandet. Studier $i$ hundra års skolpropaganda, Bonniers, 1969, voir en particulier le chapitre «Läseböckernas kör», p. 217.

4. - Nordlinder Eva, Sekelskiftets svenska konstsaga och sagodiktaren Helena Nyblom Bonniers, 1991, p. 35 .

5. - Kåreland Lena \& Werkmäster Barbro, Möte med bilderboken, Liber, 1985, p. 47. Un album était rarement tiré à plus de 2000 exemplaires tandis que les publications de Noël tiraient à environ 200000 exemplaires.

6. - Balzamo Elena, Le conte littéraire Suédois, Université de Lille III, 1987.

7. - Nordlinder Eva, Sekelskiftets svenska konssaga och sagodiktaren Helena Nyblom, 1991, p. 19. Le chercheur danois Søren Christensen observe dans Tørnerose i eventyrskoven, Gyldendal, 1976, p. 73 que la légende qui prend souvent comme cadre la vie quotidienne est fondamentalement pessimiste. Avec la légende la tragédie fait son entrée dans le monde des contes.

8. - Eva Nordlinder souligne dans son ouvrage (cf. note 4), p. 63, le grand nombre de femmes parmi les auteurs de contes et l'importance du conte comme genre féminin.

9. - Nathan Kurt Caesar, The «Kunstmaerchen»: Theory and practice of a genre variation and significance from the Goethe period to the present, 1973, voir chapitre 1.

10. - Nordlinder Eva, Sekelskiftets svenska konstsaga och sagodiktaren Helena Nyblom, 1991, p. 15.

11. - Ibid., p. 46.

12. - Oterdahl Jeanna, Den bergtagnas dotier, Folkskolans barntidnings förlag, 1922, p. 6.

13. - Ibid., p. 15.

14. - Kåreland Lena, Gurli Linders barnbokskritik, 1977, p. 69.

15. - Gehrling von Kristin, «Die Dichtung Jeanna Oterdahls», dans Studien zur Jugendliteratur. Herausgegeben von Karl Langosch, heft 4, 1958.

16. - Edström Vivi, Barnbokens form, Stegelands förlag, 1980, p. 29 et 33.

17. - Oterdahl, Jeanna. Den bergtagnas dotter, 1922, p. 33.

18. - Ibid., p. 41 et 42.

19. - Un conte intéressant de ce point de vue est «Den blödande svanen» («Le cygne blessé ») dans Den bergtagnas dotter, 1922, qui raconte la libération d'un prince transformé en cygne par un chevalier. Oterdhal utilise ce thème classique en le remaniant. Le voyage du chevalier Botvid pour trouver le cygne symbolise la transformation de la 
personnalité du héros. Il apprend à se libérer de la crainte et du doute, et, ce qui est le plus difficile encore, à s'oublier soi-même. Ce n'est que lorsqu'il est prêt à sacrifier sa propre vie qu'il réussit à libérer le prince. Le conte illustre ainsi la leçon biblique selon laquelle celui qui perd sa vie la gagne.

Le même thème revient un peu différemment, dans «Skattväktarne» («Les gardiens du trésor ») dans Sagor och visor (Contes et chansons) 1920. Un garçon vend son âme pour que sa mère guérisse et échappe à la pauvreté. Ce conte est une variante du conte populaire nordique «Prins Hatt under jorden».

20. - Oterdahl Jeanna, Den bergtagnas dotter, 1922, p. 66.

21. - Pendant la deuxième moitié du XIX ${ }^{\mathrm{e}}$ siècle, la Suède connut une forte émi-gration vers les Etats-Unis. Les courants nationalistes et romantiques de l'époque ont lutté contre cette émigration, au nom de la fidélité à la patrie et de l'attachement à la terre natale.

22. - Oterdahl Jeanna, Den bergtagnas dotter, 1922, p. 69.

23. - Dans la croyance populaire, le lutin est un être souterrain qui influe sur le destin des hommes.

24. - De même, dans d'autres contes, Oterdhal décrit une rencontre décisive qui modifie le cours de la vie d'un homme et entraîne une transformation de son caractère. C'est par exemple le cas dans «En riddare red fram...» dans Backsippor (Des coquerelles), 1917 : une pauvre bergère dont la vie est dirigée par la satisfaction des besoins élémentaires rencontre un chevalier en route pour la Terre Sainte. Le chevalier éveille en elle la conscience des valeurs spirituelles, et efface le côté grossier et terre à terre de sa personnalité.

Le conte ambigu «Djupets barn» («L'enfant des profondeurs») dans Sagor och visor (Contes et chansons), 1920, traite également de l'opposition entre l'esprit et la nature. Oterdahl développe ici la philosophie de Schelling, en particulier sa conception de la vie organique et la notion de la libération des formes de vie élémentaires. Le conte parle d'une jeune fille paralysée qui vit en contact intime avec la nature. Elle parvient à ranimer l'esprit d'un étang, un garçon à la queue de poisson et aux doigts palmés. Il s'humanise au fur et à mesure qu'il fréquente la jeune fille, mais il lui manque toujours une âme : il ne peut pas pleurer et ne sait pas distinguer le Bien du Mal. Mais, grâce à leur amour le garçon est libéré du sortilège. Cependant, c'est la mort qui réalise leur libération ultime dans ce conte mélancolique. La jeune fille n'est plus paralysée, le garçon a perdu sa queue de poisson, la dernière chose qui le liait à la boue. Accompagné de la jeune fille il peut à présent rejoindre les demeures immortelles.

25. - Bettelheim Bruno, Sagans förtrollade värld. Folksagornas inebörd och betydelse. AWE/Gebers, 1979, voir en particulier l'Introduction.

26. - Nordlinder Eva, Seketskiftets konstsaga och sagodiktaren Helena Nyblom, 1991, p. 83.

27. - Miller Alice, I begynnelsen var uppfostran, Wahlström \& Widstrand, 1983.

\section{RÉSUMÉS}

Parmi de nombreuses femmes écrivains suédoises, Jeanna Oterdahl apporta, pendant les premières décennies du XXe siècle, une contribution importante à la littérature enfantine. Elle était professeur, écrivait des livres, prononçait des conférences et publiait un grand nombre de recueils de contes. Elle fut l'une de ces nombreuses femmes cultivées, issues de la bourgeoisie qui firent de la littérature enfantine leur domaine de prédilection. Vers 1900 la littérature enfantine 
en Suède connaît son premier essor. Le prestige du conte littéraire était grand, c'était le genre que le plus d'enfants, indépendamment de leur origine sociale, avaient l'occasion de rencontrer. Oterdahl publia entre 1910 et 1961 une quinzaine de recueils de contes. La composition de ses contes est resserrée et présente la même simplicité de structure que le conte populaire. Ils traitent symboliquement des problèmes d'ordre existentiel ou moral et montrent comment se trempe le caractère du héros et comment se développe sa personnalité. Le message des contes est conforme au code moral en vigueur.

Le conte «Den bergtagnas dotter» (la fille de la femme ensorcelée) qui est construit sur le thème de l'enlèvement et celui de l'enfant substitué, cache sous l'intrigue un drame spirituel. C'est un conte moral sur l'amour, singulièrement l'amour maternel. Le thème religieux est dominant comme dans de nombreux autres contes. «Den hemlighetsfulla trädgården» (Le jardin secret) est une allégorie sur le but et le sens de la vie humaine. Le message transmis est l'idée chrétienne du sacrifice. Dans d'autres contes on note un lien évident avec une problématique d'actualité comme dans «Kvistvàttens knyte» (Le baluchon du lutin de Kvisten) où il s'agit de l'émigration, objet de vives controverses à l'époque. Le conte illustre le conflit moral d'une jeune personne et traite aussi du rapport parents-enfants. Le héros qui quitte son foyer pour aller chercher le bonheur dans le monde - un thème fréquent dans le conte populaire - est considéré ici d'un autre point de vue. Dans les contes d'Oterdhal la frontière entre la littérature enfantine et la littérature pour adultes est souvent transgressée et les textes offrent une lecture à plusieurs niveaux.

Die Schwedische Schriftstellerin Jeanna Oterdahl (1879-1965) hat bedeutende Beiträge auf dem Gebiet der Kinderliteratur geleistet. Sie war Lehrerin und schrieb mehrere Bücher für Kinder und Erwachsene, u.a. viele Märchensammlungen. Sie war eine der vielen gut ausgebildeten Frauen aus bürgerlichem Milieus, die das Kinderbuch $\mathrm{zu}$ ihrem besonderem Gebiet machten. Die schwedische Kinderliteratur kannte eine wichtige Periode $\mathrm{zu}$ Beginn dieses Jahrhunderts. Besonders interessierte man sich für das Märchen, mit welchem alle Kinder aus den verschiedenen Klassen der Gesellschaft in Kontakt kamen. Oterdahl gab zwischen 1910-1961 etwa 15 Märchensammlungen heraus. Ihre Märchen sind klar strukturiert, sie weisen die einfache Struktur des Volksmärchens auf. Sie behandeln symbolisch existensielle und moralische Probleme und schildern, oft allegorisch, die Entwicklung des Charakters.

Das Märchen «Den bergtagnas dotter» (Die Tochter der Verzauberten), das von dem Motiv Zauber und Kindertausch ausgeht, handelt, unter der äusseren, spannenden Handlung, von einem seelischen Drama. Es ist eine Geschichte von der Macht der Liebe, besonders der Mutterliebe. Das religiöse Motiv ist auffallend in diesem Märchen wie auch in vielen anderen. «Den hemlighets-fulla trädgården» (Der geheimnisvolle Garten) ist eine Allegorie von dem Ziel und der Meinung des menschlichen Lebens. Das Märchen drückt den christlichen Opfergedanken aus und vermittelt auch eine interessante Auffassung der Liebe. In anderen Märchen findet man aktuelle Anspielungen wie in «Kvist-vättens knyte» (Das Bündel des Trolles), das die Emigration behandelt. Dieses Märchen beleuchtet den Konflikt eines jungen Menschen und die Beziehung zwischen Kindern und Eltern. Hier ist das Motiv des Volksmärchens, - der Held, der in die Welt hinausgeht, - aus einer anderen Perspektive gesehen. In den Märchen von Jeanna Oterdahl ist oft die Grenze zwischen Kinderliteratur und Literatur für Erwachsene überschritten. Ihre Texte bieten die Möglichkeit einer mehrschichtigen Lektüre.

\section{AUTEURS}

\section{LENA KÅRELAND}

Institut du livre pour enfants, Stockholm 